\title{
Similarity in evoked responses does not imply similarity in macroscopic network states across tasks
}

\section{Title Page}

Abbreviated title: Dissimilar networks from similar brain responses

Author Names and Affiliations: Javier Rasero ${ }^{1,2}$, Richard Betzel ${ }^{3}$, Amy Isabella Sentis ${ }^{2,4}$, Thomas E. Kraynak $^{4,5}$, Peter J. Gianaros ${ }^{4,5}$, Timothy Verstynen ${ }^{1,2,4}$.

1: Department of Psychology, Carnegie Mellon University, Pittsburgh, PA 15213, USA

2: Neuroscience Institute, Carnegie Mellon University, Pittsburgh, PA 15213, USA

3: Department of Psychological and Brain Sciences, Indiana University, Bloomington, IN 47405, USA

4: Center for the Neural Basis of Cognition, University of Pittsburgh and Carnegie Mellon University, Pittsburgh, PA 15213, USA

5: Department of Psychology, University of Pittsburgh, Pittsburgh, PA 15260, USA

Number of pages: $29+5$ (supporting information)

Number of figures: $7+5$ (supporting information)

\section{Number of Words}

Abstract: 203

Significance Statement: 117

Introduction: 594

Discussion: 1143 
Conflict of interest statement: The authors declare no competing financial interests.

Acknowledgements: Research reported in this publication was supported by the National Heart, Lung, snd Blood Institute of the National Institutes of Health under Award Numbers P01 HL040962 and R01 HL 1089850. The content is solely the responsibility of the authors and does not necessarily represent the official views of the National Institutes of Health. 


\begin{abstract}
There is an ongoing debate as to whether cognitive processes arise from a group of functionally specialized brain modules (modularism) or as the result of a distributed nonlinear process (dynamical systems theory). The former predicts that tasks that recruit similar brain areas should have an equivalent degree of similarity in their connectivity. The latter allows for differential connectivity, even when the areas recruited are largely the same. Here we evaluated both views by comparing activation and connectivity patterns from a large sample of healthy subjects $(\mathrm{N}=242)$ that performed two executive control tasks, color-word Stroop task and Multi-Source Interference Task (MSIT), known to recruit similar brain areas. Using a measure of instantaneous connectivity based on edge time series as outcome variables, we estimated task-related network profiles as connectivity changes between incongruent and congruent information conditions. The degree of similarity of such profiles at the group level between both tasks was substantially smaller than their overlapping activation responses. A similar finding was observed at the subject level and when employing a different method for defining task-related connectivity. Our results are consistent with the perspective of the brain as a dynamical system, suggesting that task representations should be understood at both node and edge (connectivity) levels.
\end{abstract}

\title{
Significance Statement
}

There exist two contrasting views of the brain that yield different predictions of how cognition is represented. In the modular view, similar cognitive processes should have similar connectivity profiles. In contrast, a dynamical systems view allows for multiple network configurations in these situations. Here we tested both views using two tasks, color-word Stroop and Multi-Source Interference Task (MSIT), that evoke a similar brain response. Using a novel approach of instantaneous connectivity we first estimated the connectivity changes during both tasks and subsequently found that their similarity was substantially smaller than the patterns of brain activation. These findings reinforce the view of brain as a dynamical system and shed light onto how cognitive processes may be quantitatively modeled. 


\section{Introduction}

The idea of a modular mind (Fodor, 1983), where cognition arises from the interplay between specialized, domain-specific units that represent fundamental cognitive processes, has dominated the cognitive neuroscientific view of the brain since the its inception (e.g., Posner et al., 1988). Here the cognitive "modules" are mapped to unique brain areas that execute specific processes (e.g., sound frequencies, visual edges, information, muscle contractions) (Feinberg and Farah, 2006). Over the last three decades, this modular view of the brain has largely been justified from empirical observations using non-invasive brain imaging methods, like positron emission tomography (PET) and functional MRI (fMRI), where experiments and analytical methods (Friston et al., 1994) were explicitly designed to isolate clusters of regions aligned to certain functional domains, such as vision (e.g., Bihan et al., 1993), control (e.g., Porro et al., 1996), language (e.g., Binder et al., 1997), or affect (e.g., Anders et al., 2004). A critical assumption of this modularist perspective is that task-relevant representations are strictly limited to the domain-specific modules (e.g., specific brain regions), with communication between modules simply being a matter of relaying information from one stage of processing to the next. In other words, co-activating the same two modules (i.e., brain regions), in the same way across two different tasks, will also lead to similar connectivity patterns between those modules.

This picture of a modular brain has been progressively challenged over the years by the idea that cognition arises from a dynamical system. From this perspective, a given cognitive function cannot be solely understood by investigating its components separately, but requires also understanding the interactions between units as well (Gelder, 1995). While almost as old as the modularist view of the brain, this dynamical systems perspective has gained ground over the past decade in systems neuroscience, where multi-unit recording studies have shown that task representations emerge as a low-dimensional manifold of population activity, both within and between brain areas (Russo et al., 2020; Churchland et al., 2012; Sadtler et al., 2014; Oby et al., 2019). This observation at the microscale level extends to observations of macroscopic brain dynamics as well (e.g., Kriegeskorte et al., 2008; Ejaz et al., 2015). Indeed, with the rise of connectomics (Behrens and Sporns, 2012), the idea of the brain as a dynamical network (Sporns, 2013), where information is also encoded between units (Crossley et al., 2013; Yeo et al., 2014; Bertolero et al., 2015), has gained popularity in cognitive neuroscience. A contrasting assumption of the dynamical systems view, compared to the 
modularist view, is that task-relevant representations are encoded by both the individual regions and the communication dynamics (i.e. connectivity) between those regions. Therefore, two tasks may activate the same pattern of nodes, but express dissimilar connectivity profiles (Prinz et al., 2004; Hooper, 2004).

Here we sought to shed light on this debate by testing whether the apparent overlapping patterns of BOLD activation that are elicited during two response conflict tasks (Sheu et al., 2012), the color-word Stroop task (Stroop, 1935) and the Multi-Source Interference Task (MSIT) (Bush and Shin, 2006), also have similar task-related connectivity profiles. In a sample of neurologically healthy adults $(\mathrm{N}=242)$, we first computed their instantaneous functional connectome, using a novel approach that temporally unwraps Pearson correlations to generate time series along edges, representing the inter-node BOLD signal co-fluctuations (Zamani Esfahlani et al., 2020). Then, by means of a general linear model (GLM), we assessed the task-based contributions to the edge time series, quantifying the amount of out-of-sample variability that they contained. We then compared the degree of between-task similarity at the regional activation and connectivity levels.

\section{Methods and Materials}

\section{Participants}

We analyzed task and resting-state fMRI data from the Pittsburgh Imaging Project (PIP), which is a registry of behavioral, biological and neural correlates of cardiovascular disease risk among otherwise healthy community-dwelling adults (aged 30-54 years). Details of this project can be found in the supplementary material of Gianaros et al. (2020). We selected a subset of 242 subjects (female $=119$, mean age $=40 \pm 6$ years, min age $=30$ years, max age $=51$ years) that had full temporal and spatial coverage and exhibited low average motion (mean framewise displacement, estimated using the method in Power et al., 2012, lower than $0.35 \mathrm{~mm}$ ) across the three fMRI tasks used in our study.

\section{MRI Data Acquisition}

MRI data were acquired on a 3 Tesla Trio TIM whole-body scanner (Siemens, Erlangen, Germany), equipped with a 12-channel head coil. Functional blood-oxygen-level-dependent (BOLD) images were acquired from a T2*-weighted gradient echo-planar imaging sequence (repetition time $=2000 \mathrm{~ms}$, echo time $=28$ 
$\mathrm{ms}$; field of view $=205 \times 205 \mathrm{~mm}$ (matrix size $=64 \times 64$ ), slice thickness $=3 \mathrm{~mm}$ (no gap); and flip angle $=90^{\circ}$ ). For anatomical coregistration of the fMRI images, a high-resolution T1-weighted image per subject was also acquired $\left(\right.$ MPRAGE, repetition time $=2100 \mathrm{~ms}$, echo time $=3.29 \mathrm{~ms}$, inversion time $=1100 \mathrm{~ms}$, flip angle $=8^{\circ}$, field of view=256 $\mathrm{mm} \times 208 \mathrm{~mm}$ (matrix size: $256 \times 208$ ), slice thickness=1 mm with no gap).

\section{Tasks}

We used two tasks that involved processing conflicting information and response inhibition. Both tasks consisted of 4 blocks that defined a congruent information condition, interleaved with 4 blocks of trials where the participant received incongruent information. Both task conditions had a duration of 52-60 secs, and were preceded by a variable 10-17 sec fixation block. It total, each task had a duration of 9 min and 20 secs.

In the color-word Stroop task, participants had to select 1 of 4 identifier words using a response glove (e.g., thumb button 1 = identifier word on the far left, etc.), such that its name indicates the color of target words located in the center of a screen. During the congruent trials, the four identifier words were all in the same color as the target words. Instead, in incongruent trials, identifier words had all different colors, and the option to select was in a color incongruent with the target words. This kind of task usually evokes a brain response that activates regions in the anterior insula, parietal cortex, basal ganglia, thalamus, and cerebellum; while deactivating areas belong to the so-called 'default-mode network' (see Fig. 1A, B and C).

In the MSIT, which corresponded here to a modification from the original task version (Bush and Shin, 2006), participants had to select 1 of 3 numbers such that it differed from the others 2 by pressing buttons on the glove, where each button matched a number on the screen (thumb button $1=$ number 1, etc.). During congruent trials, targets' position matched that on the glove, whereas during incongruent trials this position did not match the glove's button location. This task elicits a brain pattern response that is largely similar to that in Stroop task (see Fig. 1D, E and F and Sheu et al., 2012 for more details on the MSIT and the Stroop task).

In incongruent conditions of both tasks, accuracy was titrated to $\sim 60 \%$ by altering intertrial intervals, i.e. consecutive accurate choices led to shortened intertrial intervals. To control for motor response differences 

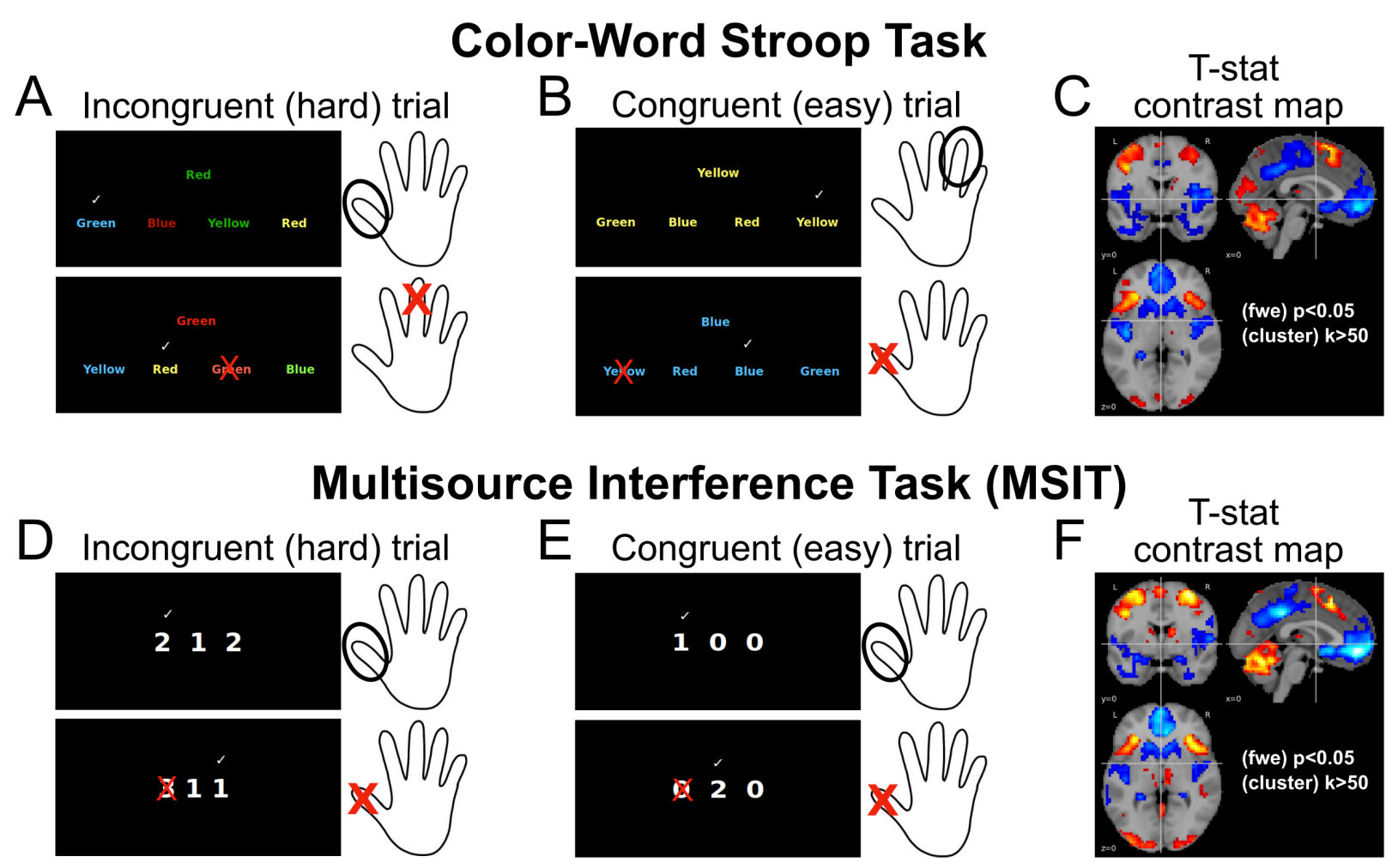

Figure 1: Stroop task and MSIT paradigms and their brain response. For both Stroop task and MSIT, illustration of incongruent (A, D) and congruent trials (B, E). Trials consisted of blocks of 52-60 s duration, interleaved with a 10-17 s fixation block. Contrasting brain activity between incongruent and congruent condition gives rise to a similar brain response $(\mathrm{C}, \mathrm{F})$.

between conditions in both tasks, the number of trials in the congruent condition was yoked to the number completed in the incongruent condition. Yoking was implemented by (1) administering an incongruent block first and (2) presenting congruent condition trials at the mean intertrial interval of the preceding incongruent block.

Finally, we also used a five-minutes resting-state scan, during which the participants were told to keep their eyes open.

\section{Preprocessing}

Data were preprocessed using the default pipeline of fMRIprep (Esteban et al., 2018), a standard toolbox for fMRI data preprocessing that provides stability to variations in scan acquisition protocols, a minimal user manipulation, and easily interpretable, comprehensive output results reporting. In order to avoid possible 


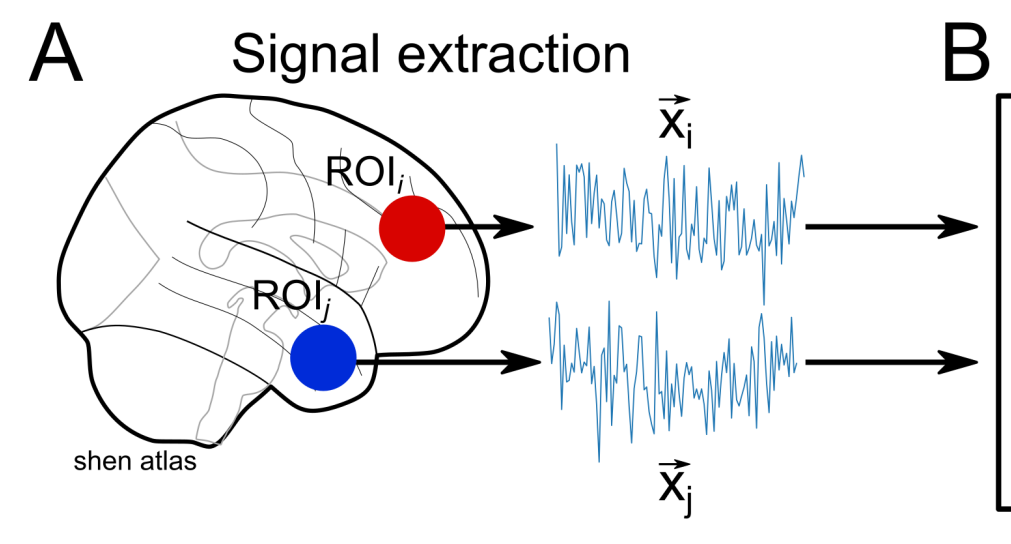

Denoise regression

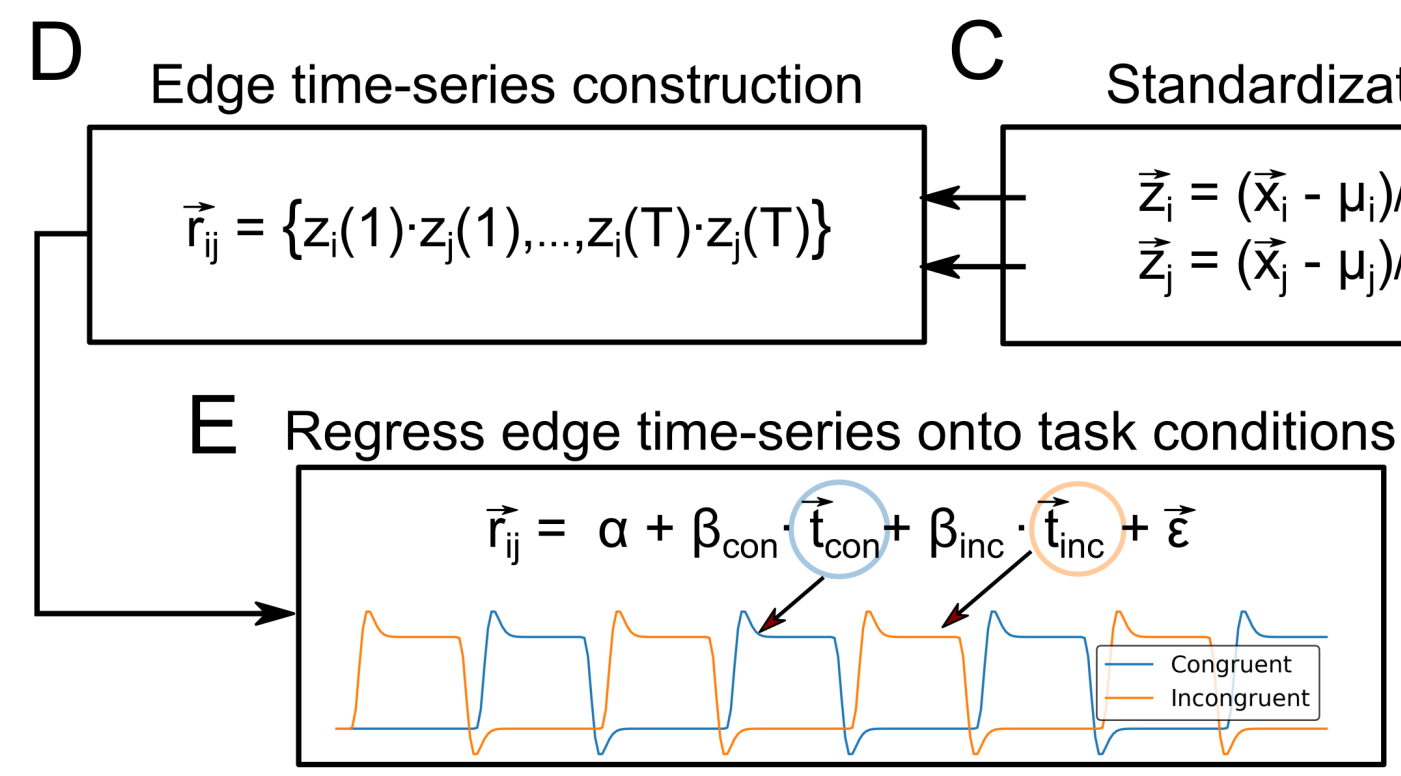

Figure 2: Estimation of intrinsic and task-related connectivity. For a given pair of regions in a parcellated atlas, the average signal within them was first computed (A). The time series were then denoised (B) and standardized (C). Subsequently, they were multiplied component-wise (D). Finally, the resulting time profile was regressed onto a design matrix to model intrinsic (intercept term) and task-related connectivity (E).

susceptibility distortions in the BOLD images triggered by field inhomogeneities, a correction step via a nonlinear registration was also applied during the preprocessing workflow ("Fieldmap-less" option in the toolbox).

\section{Connectivity (Edge)-level analysis}

We estimated task-based connectivity contributions using the edge, or co-fluctuation, time series proposed in Faskowitz et al. (2020); Zamani Esfahlani et al. (2020). A sketch of this full estimation procedure can 
be found in Fig. 2. We first (step A) reduced the spatial resolution of the minimally preprocessed time series by computing the voxel-wise average signal within each region (ROI) in a 268-parcels atlas (Shen et al., 2013). Following Finn et al. (2015), each of these regions were also identified to a specific intrinsic connectivity network (see Fig. S1). Then, let $\vec{x}_{i} \equiv\left\{x_{i}(1), \ldots, x_{i}(T)\right\}$ be the time series of $T$ scans (the fullscan sequence) for a given parcel $i$ in such atlas. Each of these parcellated time series were subsequently (step B) denoised by means of a linear regression model, in a single step that avoid artifacts from being reintroduced in the data (Lindquist et al., 2019), in order to remove effects from motion (24 parameters which included 3 translations, 3 rotations, their derivatives and the square of all these terms), the average white-matter signal, the average CSF signal, the average brain signal, periodic oscillations greater than 187 $\mathrm{s}$ (4 cosine terms) and task activations (24 terms). This last set of regressors consisted of 12 finite impulse response (FIR) terms per task condition (congruent and incongruent) to flexibly model an hemodynamic response function (HRF) of about 24 s to external stimuli and that was included so as to avoid systematic inflation of functional connectivity produced by task activations (Cole et al., 2019). The resulting denoised ROI time series were standardized (step C), i.e. $\vec{z}_{i}=\frac{\vec{x}_{i}-\mu}{\sigma}$, and then used to generate the edge time series $\vec{r}_{i j}$ (step D) as the component-wise product between pairs of standardized time series, i.e. $\vec{r}_{i j}=$ $\left\{z_{i}(1) \cdot z_{j}(1), \ldots, z_{i}(T) \cdot z_{j}(T)\right\}$. At this point, if we summed these components up and divide by $T-1$, we would obtain the Pearson correlation coefficient that usually represents the static functional connectivity between BOLD time series. That is, each edge time series can be interpreted as a temporal decomposition of a functional connection (correlation) into its framewise contributions. Instead, we continued working on these edge time series as response variables in a general linear model (step E) in order to estimate intrinsic and task-dependent connectivity responses. To this end, the input design matrix included an intercept term and a set of regressors for each task condition (congruent and incongruent), which comprised a boxcar function convolved with the usual double gamma hemodynamic response function and its temporal and dispersion derivatives.

Prior to any statistical analysis, time series in both sides of the regression model were prewhitened through a first-order autoregressive model in order to account for the temporal autocorrelations. We assumed this standard procedure for dealing with temporal autocorrelations in order to have the same statistical pipeline as that in the activation analysis (see next subsection). Future studies should investigate the most appropriate 
procedure for accounting for autocorrelations using edge time series. After this first-level estimation, taskbased connectivity changes were computed as contrasts of parameters and subsequently used to assess edgewise group-level effects by means of a one-sample t-test. Statistical inference at a usual 0.05 significance

level was finally performed, after correcting the family-wise error (Holm-Bonferroni procedure) due to multiple testing. All these statistical analyses of the edge time series were carried out using Nilearn 0.7 (Abraham et al., 2014).

To test whether or not the inferred connectivity maps reliably generalized to unseen data, the same general linear model was embedded within a k-fold cross-validation scenario. Specifically, for each edge time series used as a response variable and the task regressors (the same set of terms described previously) as predictors, the group-level $\alpha$ (intercept) and $\beta$ (slopes) were estimated as their average across the first-level estimations from the subjects in the training set. Subsequently, these were used to quantify to what degree mean edge time series from the subjects in the test set were predicted. The difference between the predicted and observed edge time series was evaluated using the coefficient of determination $R^{2}$. The advantage of using this metric is that for values greater than zero it means that the task stimuli explained a non-zero variability on top of that of the intrinsic or background signal, here modeled as the intercept. A 3-fold cross-validation was employed, where the full data were divided into 3 smaller sets ("folds"), such that for each of these (the test set) the generalization was determined using the coefficients estimated in the other 2 folds (the training set). This process was further repeated 20 times with different splitting seeds. The final performance per edge was then the average $R^{2}$ across all folds and repetitions.

\section{Activation (Node)-level analysis}

We also analyzed the preprocessed BOLD images at the node level, which involved estimating brain activation changes during the different task conditions. Such analyses are usually carried out introducing some level of spatial smoothing to the data in order to increase sensitivity. However, in order to enable a fair comparison with the edge-level results, no smoothing was performed and instead, brain activations were estimated using the same parcellated BOLD time series.

To this analysis, we employed again a GLM with the parcellated BOLD time series as response variables and a design matrix that included the same set of task regressors used with for the edge-wise analyses, as 
well as the same covariates that were regressed out prior to this, i.e. the 24 motion parameters (Friston et al., 1996), the cosine terms to account for oscillation effects greater than $187 \mathrm{~s}$, the average signal within white-matter tissue, the average signal within CSF tissue, and the average signal within the whole-brain. We considered this last regressor, not common in brain activation analyses, for consistency again with the edge-level analyses (see previous section). Group-level effects were similarly assessed using a one-sample t-test.

\section{Generalized Psychophysiological Interaction}

We compared the connectivity analysis using the aforementioned edge time series approach with a model of Generalized Psychophysiological Interactions (PPI), which is a standard approach for estimating taskdependent functional connectivity changes (McLaren et al., 2012) and is based on a general linear model of task-moderated temporal association between pairs of brain units. Specifically, for a given pair of BOLD time series $\vec{x}_{i}$ and $\vec{x}_{j}$, such model includes one of them as the response variable and as inputs the other BOLD time series, the group of task regressors, the interaction terms between these task regressors and the input BOLD time series, and the possible confounders to consider in the model. In our case, the generalized PPI model can be written as follows:

$$
\vec{x}_{i}=\alpha+\beta_{i j}^{b c k} \cdot \vec{x}_{j}+\beta^{\text {task }} \times \mathcal{T}+\beta_{i j}^{p p i} \times \mathcal{I}_{i}+\beta^{c o v} \times \mathcal{C}+\vec{\epsilon},
$$

where $\mathcal{T}$ is a matrix whose columns are the HRF convolved box-car congruent and incongruent time profiles and their derivatives and dispersion terms, $\mathcal{I}_{i}$ the matrix with the PPI terms from each condition, i.e. the interaction term between each task condition's time profile and the input time series $\vec{x}_{i}$, and $\mathcal{C}$ a matrix with the different covariates to include in this model, which in our case comprised the 24 motion parameters, the average white-matter signal, the average CSF signal, the average brain signal and cosine expansion for a $187 \mathrm{sec}$ high-pass filtering. Once all the parameters in this model were estimated, task-based connectivity changes were evaluated by contrasting the incongruent and congruent PPI terms and their effect at the group level assessed using a one-sample t-test. In this way, a matrix of estimated task-based connectivity changes can be constructed. However, since a PPI model yields non-symmetrical matrices, we symmetrized them by averaging their corresponding upper and lower triangular elements as done in Di et al. (2017), which 
enabled a direct comparison with the connectivity profiles obtained from the edge time series approach.

\section{Results}

\section{Group-level activation profiles}

We begin by noting that the Stroop task and MSIT are effortful cognitive control tasks that are known to evoke similar spatial patterns of activation across the brain, particularly the neocortex (see contrasts maps in Fig. S2 and in Sheu et al., 2012). For our dataset such similarity at the region-level (Voxel-wise estimations with comparable spatial smoothing as the regions yielded similar values) is quantified by a Spearman correlation coefficient, $\rho$, between un-thresholded incongruent-vs-congruent t-stat maps. This $\rho$ was equal to 0.87 , and a Dice similarity coefficient (DSC) equal to 0.86 , after binarizing these maps as to whether their t-stats rejected or not the null hypothesis at $\alpha=0.05$ after family-wise error correction. As shown in Fig. S2, increases in brain activity in incongruent trials, with respect to congruent trials, were located in areas typically engaged during the processing of conflict information and response inhibition, such as the anterior cingulate cortex, anterior insula, parietal cortex, basal ganglia, thalamus, and cerebellum. In contrast, de-activations took place in areas within the ventromedial prefrontal cortex, perigenual anterior cingulate cortex, posterior cingulate cortex, and precuneus, which all comprise the default-mode network. As a consequence, these results show that similar cognitive contexts evoke similar patterns of activity outputs in areas segregated across the brain.

\section{Exploration of co-fluctuating brain activity}

For illustrative purposes, we examined the task-related effects on the inter-region co-fluctuations by computing the root sum of squares (RSS) across edges at each time frame. It is important to clarify that, for this calculation, parcellated BOLD time profiles prior to edge time series formation included all task events, in contrast to subsequent analyses. As shown in Fig.3A and B, during both tasks high moments of cofluctuations tended to be synchronized across subjects, concentrated mostly around the rest periods separating congruent and incongruent block conditions. In both the congruent and incongruent blocks, there appears to be a consistent reduction in global connectivity, with sporadic and inconsistent periods of brief synchronous activity that qualitatively appear more frequent during incongruent blocks. 

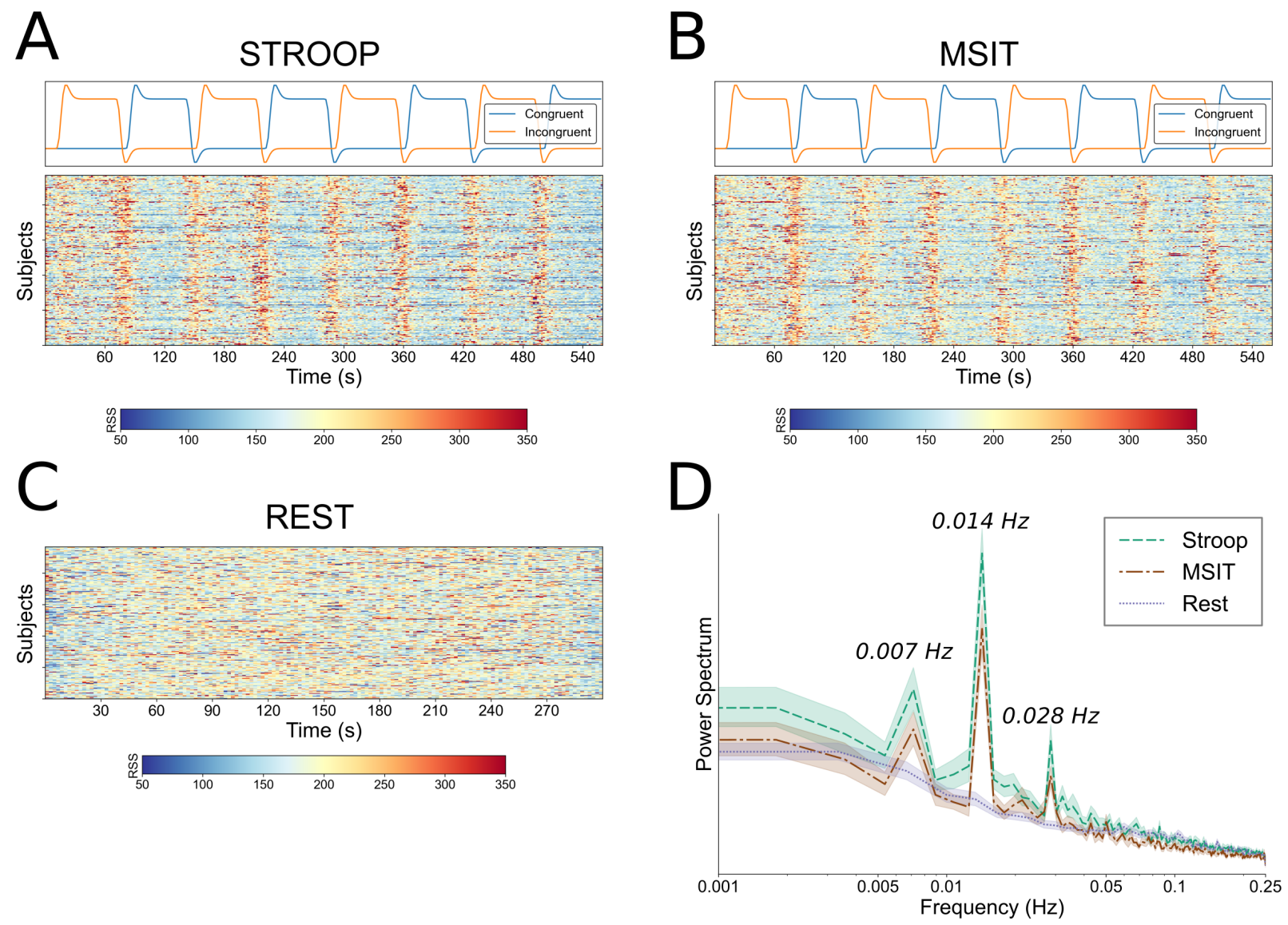

Figure 3: Analysis of the root sum of squares time profiles. For each subject, the root sum of squares of the edge time series that include the task effects for Stroop task (A), MSIT (B) and resting-state acquisiton (C). Their power spectrum (in arbitrary units) using a periodogram (D), averaged across subjects.

In contrast to the task patterns, for the resting-state run, where no external stimulus was presented, we did not see evidence of between-subjects synchronization of high amplitude co-fluctuations (Fig. 3C). Though the overall presence of these brief co-fluctuations appears to be more frequent in the resting-state run than during either of the two tasks. These results were further confirmed by inspecting the subject-averaged power-spectrum of the RSS for the three tasks (Fig. 3D). For both Stroop and MSIT, there was an overall increase in power at frequencies consistent with task onsets and offsets.

\section{Group-level connectivity profiles}

We estimated task-related connectivity using a GLM on the edge time series. Three coefficients (i.e., intercept, congruent, and incongruent) for both Stroop task and MSIT were estimated for each edge, while 


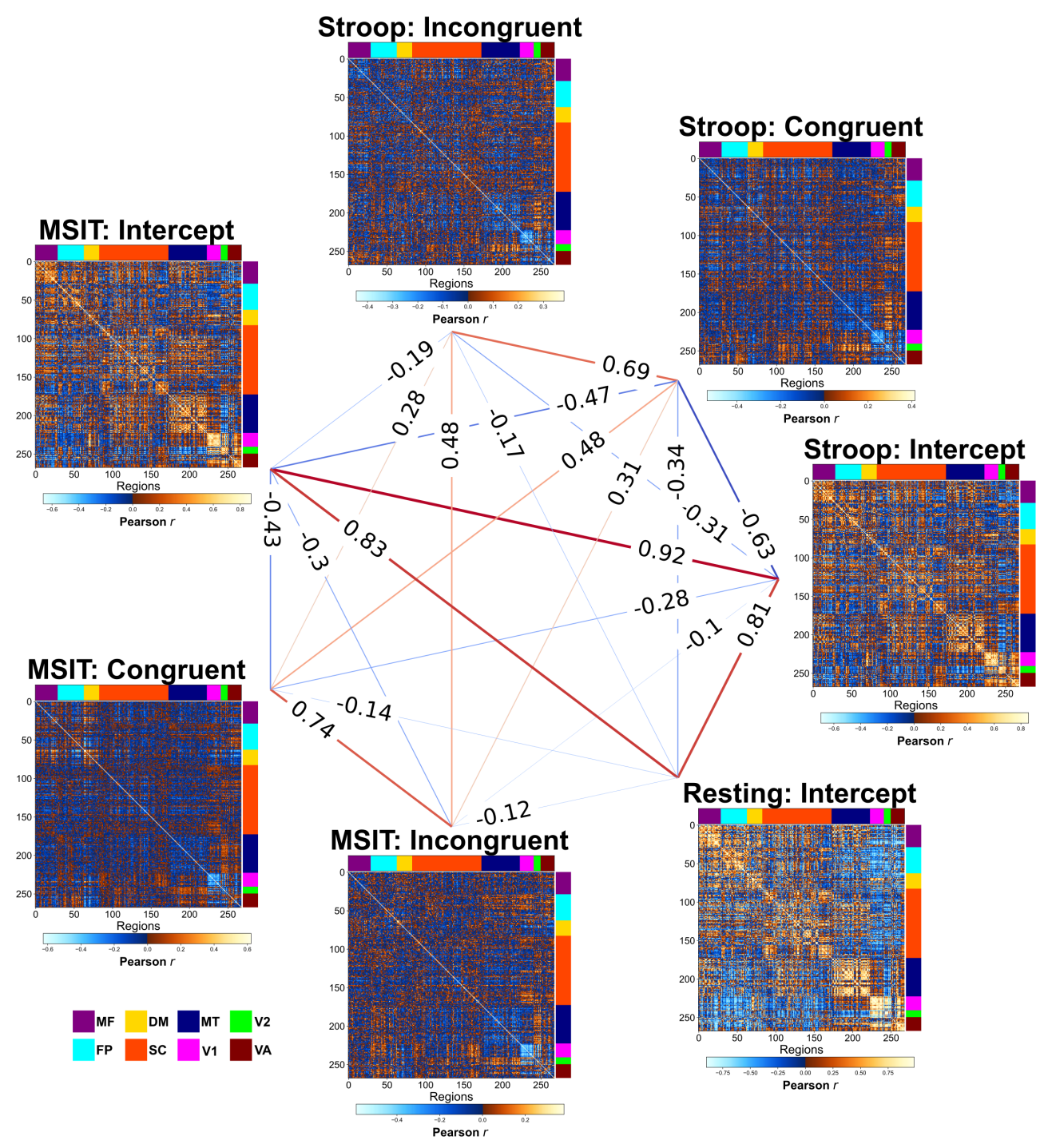

Figure 4: Connectivity patterns at the group level. For Stroop task, MSIT and resting-state connectivity matrices using the intercept, congruent and incongruent GLM estimations at the group level. Regions (i.e. the rows and columns) have been arranged based on their belonging to an intrinsic connectivity network (see Methods). In the middle in the form of a graph, the Spearman correlations between the upper-triangular elements of these matrices. MF: Medial-Frontal; FP: Frontoparietal; DM: Default-mode; SC: SubcorticalCerebellum; MT: Motor; V1: Visual-1; V2: Visual-2; VA: Visual-Association.

for resting state a single coefficient per edge was obtained (i.e., intercept only). The resulting group-level connectivity profiles are displayed in Fig. 4, where the t-stats for each of these coefficients were converted to correlations using the transformation $r^{2}=\frac{t^{2}}{t^{2}+N-1}$, with $\mathrm{N}$ being the number of subjects.

The first thing to note is that, after accounting for condition effects during the two tasks, we were able to 
recover the intrinsic brain networks observed during resting state. The intercept profiles for both Stroop and MSIT had a high degree of similarity to the resting state profile ( $\rho=0.81$ and $\rho=0.83$ respectively), as well as a high degree of similarity to each other $(\rho=0.92)$.

On the other hand, a largely different profile emerges during congruent and incongruent conditions in both tasks. These networks showed much lower overall connectivity, and a shift towards more negative connectivity values, than the intercept profiles. Despite this difference from the intrinsic networks, the conditionrelated profiles (i.e. congruent and incongruent) had a decent degree of within-task similarity ( $\rho=0.69$ for Stroop and $\rho=0.74$ for MSIT), demonstrating that both conditions recruit largely consistent networks overall. Less similarity was observed between-tasks profiles, whether it be using within condition comparisons ( $\rho=0.48$ in both cases), or between-condition comparisons (Stroop Congruent-MSIT incongruent $\rho=0.31$, Stroop Incongruent and MSIT Congruent $\rho=0.28$ ).

Taken together, these results confirm that our method was able to reliably characterize both task and intrinsic (resting) networks, at the group level, using the edge time series method.

\section{Patterns of connectivity changes between task conditions}

The network profiles that emerged as a consequence of conflict processing (i.e., during incongruent blocks, relative to congruent blocks) were quantified at the group level by contrasting subject-level connectivity estimations from both task conditions. The resulting incongruent-vs-congruent statistical maps for both tasks are displayed in Fig. 5 (Left plots, panels A and B), with 1228 (Stroop task) and 1076 (MSIT) edges that were significant at $\alpha=0.05$ after family-wise error correction (red colors denote a greater connectivity during incongruent trials than during congruent trials, and blue colors the opposite). In both cases, connectivity differences were primarily associated with default-mode, frontoparietal, medial-frontal and visual systems, as measured by the average significant edges per region found in those networks. Furthermore, inspecting the sign of these differences (Fig. 5, right side of panels A and B), an increased connectivity appeared to be dominated by edges connecting regions of distinct intrinsic major systems, particularly those between the default-mode and the frontoparietal and visual-association systems, and medial-frontal areas with the frontoparietal cortex. In contrast, significant decreases in connectivity during incongruent trials with respect to congruent trials appeared to mainly involve regions of the same major system, specially those within the 


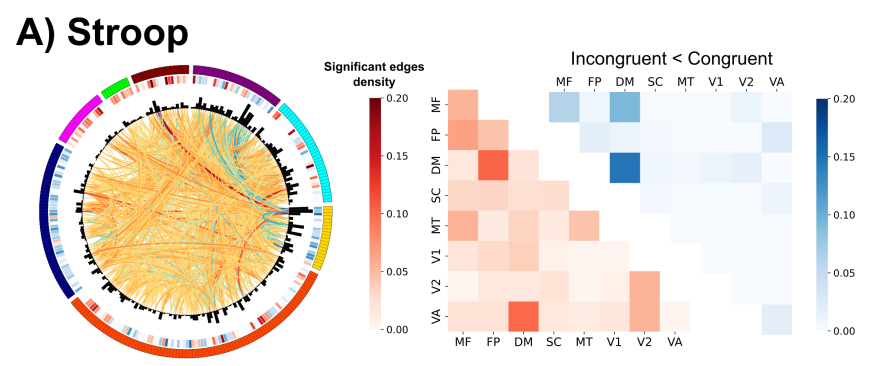

\section{B) MSIT}

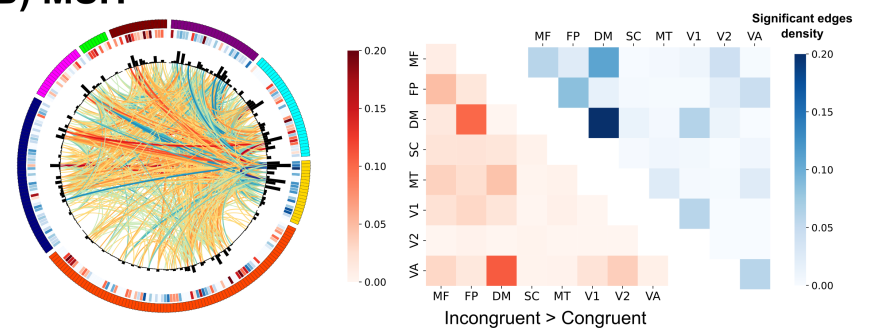

\section{C) Stroop vs MSIT}
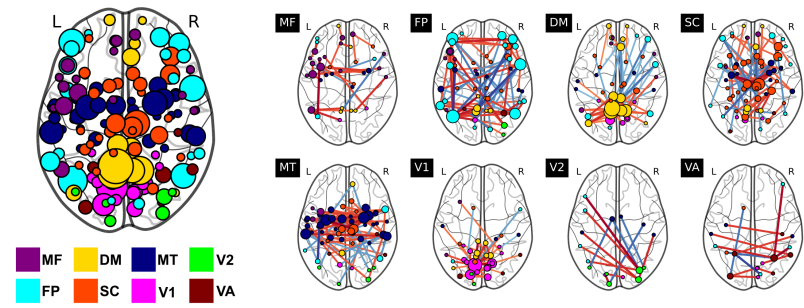

Figure 5: Incongruent-vs-congruent connectivity differences. For Stroop task (A) and MSIT (B), on the left side and from outer to inner circular plots display each regions arranged and colored based on their intrinsic connectivity network identification, their incongruent-vs-congruent group-level $t$-stat from brain activity, their node degree from the incongruent-vs-congruent significant edges and finally the t-stat of these edges (red: incongruent $>$ congruent, blue: incongruent $<$ congruent). At both node and edge-level, only significant results (at $\alpha=0.05$, Bonferroni corrected) are shown. On the right side the number of significant edges within and between intrinsic connectivity networks, normalized by the total number of edges in each case. (C) Using the significant edges from a paired t-test at $\alpha=0.05$ Bonferroni corrected, between-tasks differences in incongruent-vs-congruent connectivity in node degree (left panel) and the edge t-stat values to regions of each intrinsic connectivity network (right panel). MF: Medial-Frontal; FP: Frontoparietal; DM: Default-mode; SC: Subcortical-Cerebellum; MT: Motor; V1: Visual-1; V2: Visual-2; VA: VisualAssociation.

default-mode and medial frontal networks.

However, despite the apparent degree of similarity in conflict processing between the two tasks, the Stroop and MSIT also exhibited important connectivity differences. For example, concentrating on the $10 \%$ of edges with the largest absolute t-stat values $(n=358)$, the Stroop task contained a significantly greater number of positive (i.e. increased connectivity during incongruent trials) to negative (i.e. decreased connectivity 
during incongruent trials) edges than the MSIT (Fisher exact's test, odds ratio $=3.76, p=4.00 \times 10^{-14}$ ). On the other hand, a paired-sample t-test performed on individual edges revealed that these between-task network differences spanned the entire brain (see Fig. 5C), though they prominently expressed in the dorsolateral prefrontal and posterior parietal cortex, both responsible for executive function, as well as in the posterior cingulate cortex, that is strongly implicated during control processes, and the primary visual cortex. As a consequence, these results suggest that the Stroop task and MSIT have substantial differences in their network profiles.

\section{Reliability of edge-wise responses}

We explored the reliability of connectivity maps shown so far by testing whether edges could be predicted out-of-sample by the tasks. Since we had two fMRI tasks (Stroop and MSIT), this gave rise to two possible predictive scenarios: "within-tasks", if out-of-sample predictions were performed on the same task used for training, or "between-tasks", if out-of-sample performance was tested on the task not used for training. The edge-wise prediction rates are displayed in Fig. 6A and B, contrasting within- and between-task performance for both Stroop and MSIT data used as the training set. First, it is noteworthy that approximately $88 \%$ (Stroop) and 94\% (MSIT) of the significant edges resulting from the incongruent-vs-congruent contrast reported in previous sections could be predicted here within-tasks, demonstrating the reliability of these connectivity patterns. Second, one can see that the task-based signal at each edge varied dramatically across the entire brain network, with some edges containing more than $30 \%$ variability attributable to the task conditions. Nevertheless, across both Stroop and MSIT only less than 10\% of the edges appeared to encode meaningful task variability (i.e. average $R^{2}$ greater than zero at $95 \%$ confidence level).

More importantly, edges largely tended to generalize better within-tasks. For example, during the Stroop task 2573 edges could be predicted if tested on the same task (median $R^{2}=0.026$ across these edges), whereas between-tasks this number dropped to 998 (median $R^{2}=0.022$ across these edges). Moreover, only 474 of these edges could be predicted successfully both within and between-tasks, so the intersection between binary masks of predicted edges in both cases was low $(D S C=0.265)$. Likewise, similar values were found for the MSIT (2567 predicted edges within-tasks and median $R^{2}=0.028$ across them, 914 predicted edges between-tasks and median $R^{2}=0.016$ across these edges, intersection $D S C=0.228$ ). 


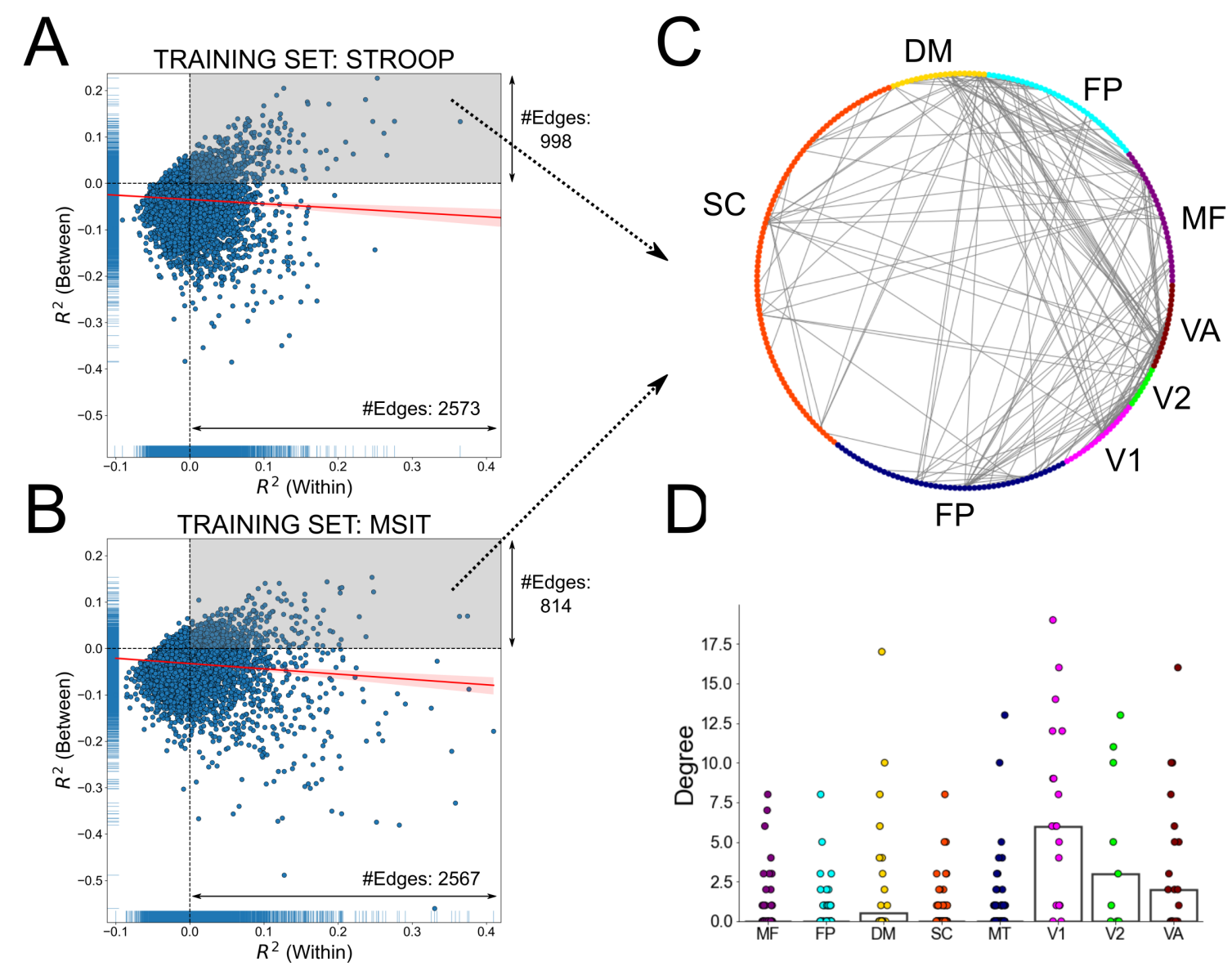

Figure 6: Edge-wise out-of-sample generalizability. The coefficient of determination for each edge predicted by the tasks regressors, using A) Stroop and B) MSIT as training set. Values on the horizontal axis represent predictions on the same task used for training (within-tasks), whereas between-tasks values (predictions on the different task used as training) lie on the vertical axis. C) Binary graph that displays the sub-network formed by those edges that could be predicted (average $R^{2}>0$ at 95\% confidence level) within and between-tasks in both MSIT and Stroop task (points in the gray area in panels A and B). D) Using the aforementioned sub-network, node degree (number of edges connected to it), grouped by intrinsic connectivity network. Each point then represents a region. The bars show the median degree across regions within each intrinsic connectivity network. MF: Medial-Frontal; FP: Frontoparietal; DM: Default-mode; SC: Subcortical-Cerebellum; MT: Motor; V1: Visual-1; V2: Visual-2; VA: Visual-Association.

Consequently, within- and between-tasks predictions had a poor correspondence (see the almost flat regression line in Fig. 6A and B), which is consistent with the differences in connectivity profiles between both the MSIT and Stroop task observed in previous sections. Interestingly, in both scenarios there was a subset of edges that were not predicted by the same task used for training, but by the other task (points in the left upper corner of Fig. 6A and B). 

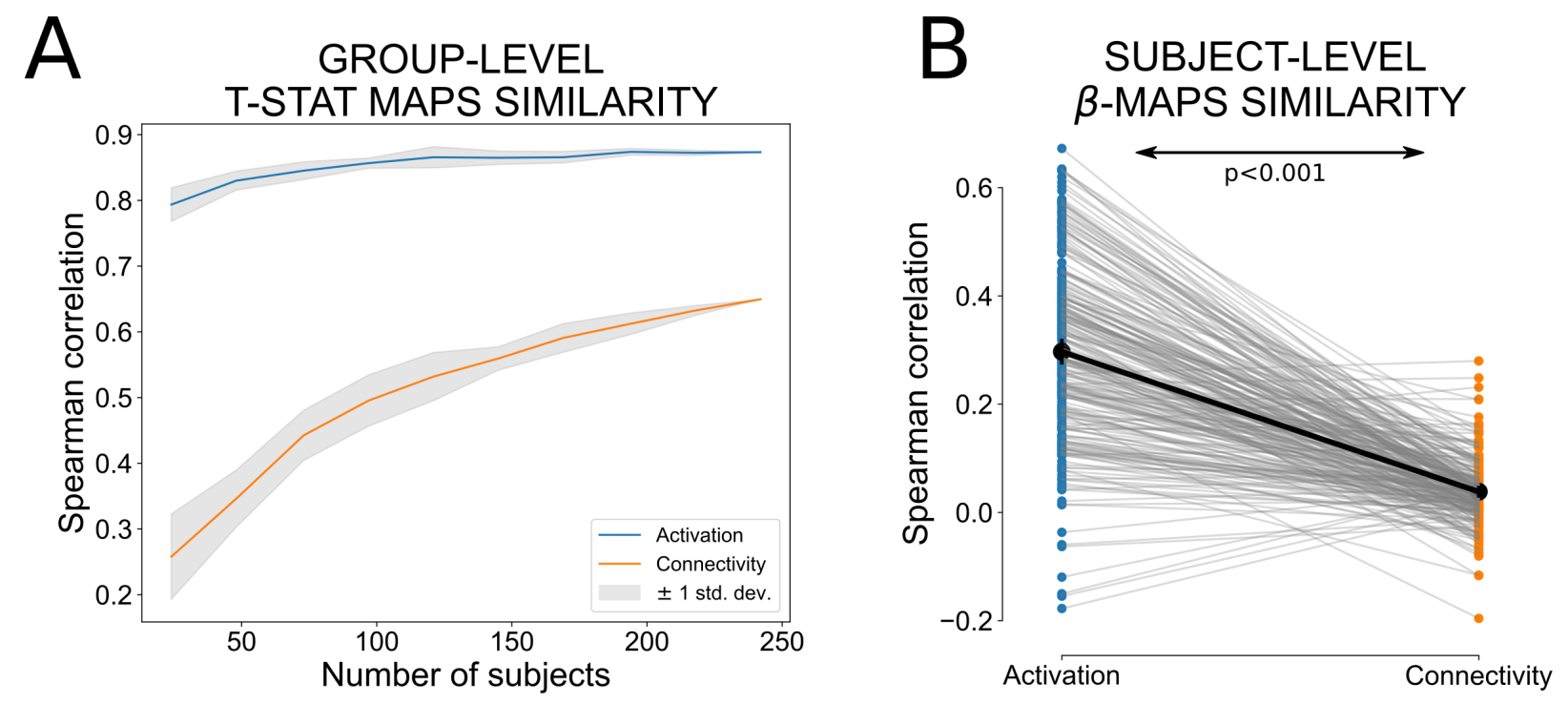

Figure 7: Between-task similarity of activation and connectivity profiles. A) Spearman correlations between tasks from the group-level t-stat incongruent-vs-congruent maps for both brain activation (blue line) and task-based connectivity (orange line), varying the number of subjects used for their estimation. Each curve represents the average similarity and the gray area the standard deviation after repeating 10 times the estimation procedure to consider different subjects. B) For each subject (a dot in the figure), the Spearman correlation between the incongruent-vs-congruent $\beta$ maps between tasks for both brain activation (blue points) and task-based connectivity (orange points). A paired t-test then quantified the statistical difference between both distributions.

Finally, we wondered which sub-network(s) appeared to be most consistent across tasks by looking at the shared edges between the Stroop and MSIT that could be predicted both within and between-tasks (points between the right upper corners of Fig. 6A and B). The resulting sub-network contained 239 edges (Fig. 6C), with a prominence of connections to areas within and between visual systems (see Fig. 6D), suggesting that part of the visual information is similarly encoded and preprocessed by both tasks, and some areas in the default-mode network.

\section{Comparison of similarities in activation and connectivity profiles between tasks}

We have previously shown that both Stroop and MSIT elicit largely overlapping patterns of brain activation $(\rho=0.87, D S C=0.86$, see also (Sheu et al., 2012)). In contrast, estimated edge-wise responses suggest that both tasks appeared to differ at the connectivity level. But is the lower similarity of network profiles between-tasks really that different than the similarity in activation profiles? The between-task similarity 
in incongruent-vs-congruent block connectivity profiles was equal to $\rho=0.64$ and $D S C=0.43$ at $\alpha=$ 0.05 , after family-wise correction, which indeed constitute a considerable reduction with respect to the aforementioned similarity rates from activation patterns.

In order to show that this reduction in similarity scores for task-related connectivity patterns was not due to correlating a larger number of features from the edges $\left(\frac{268 \times 267}{2}\right.$ edges) than in the activation maps (only 268 components, since these were also considered at the region-level), we repeated this calculation taking subsamples (number of sub-samples=10000) of 268 edges in the connectivity maps. Across all these subsets, we found similar between-tasks Spearman correlation values $(0.65 \pm 0.04$, see Fig. S4) as the one using the full network. Furthermore, this reduction became even more evident as the number of subjects decreased, and was alternatively replicated in an overlapping analysis using Dice similarity coefficients for different thresholds (see Fig. 7A).

Since the previous calculation concentrated exclusively on group-level patterns, we also tested whether the same qualitative findings were obtained using instead the subject-level estimations. Specifically, for each individual we correlated the incongruent-vs-congruent activation and connectivity $\beta$ maps of both tasks (see the sample distributions in Fig. 7B). The reason for using here the $\beta$ estimations instead of the $\mathrm{t}$-stat maps is that temporal autocorrelations in the time series produced a different number of degrees of freedom across nodes and edges in both tasks, in contrast to the group level, where the degrees of freedom remained always the same ( $N-1$, with $N$ the number of subjects). A paired t-test then showed that, as found before with the group-level maps, between-tasks similarity rates of brain activation maps were higher than those from task-based connectivity changes (Cohen's $d=2.07, p<0.001$ ).

Finally, we wondered whether the reduction in similarity between Stroop and MSIT task-dependent network profiles was influenced by the approach adopted to define connectivity. Consequently, we tested this possibility by replicating the previous analyses using a generalized Psychophysiological Interaction (PPI) model, which is a standard and common framework for assessing task modulated functional connectivity. As shown in Fig. S5, albeit small differences exist, particularly within the motor system, both approaches appeared to yield fairly similar incongruent-vs-congruent contrast network profiles in both tasks (Stroop $\rho=0.74$, MSIT $\rho=0.77)$. More importantly, these PPI-based connectivity patterns also showed a reduced similarity 
in connectivity patterns across tasks $(\rho=0.53, D S C=0.35)$ compared to what is observed in the brain activation patterns.

\section{Discussion}

Here we set out to determine whether two tasks with highly similar activation profiles also share common task-related network profiles. Using a GLM framework on instantaneous connectivity estimates (Faskowitz et al., 2020; Zamani Esfahlani et al., 2020), we were able to successfully separate task-free (intrinsic) from task-dependent network contributions, in line with the extensive evidence that task functional connectivity is jointly shaped by both intrinsic and evoked network architectures (Cole et al., 2014). Subsequently, the task-related estimations were used to generate patterns of connectivity changes across trials, which allowed us to show that network profiles exhibited a lesser degree of similarity between tasks compared to the activation profiles. These differences were largely expressed in subnetworks important for high level information processing on action selection, such as those involving the dorsolateral prefrontal and parietal cortices (Banich et al., 2000a; Milham et al., 2001; MacDonald, 2000; Bush and Shin, 2006; Deng et al., 2018), as well as the precuneus (Sheu et al., 2012). These regions in particular comprise a subnetwork that appears to be most sensitive to task-relevant information content and therefore more susceptible to change with cognitive context (Banich et al., 2000b). This difference in task-related connectivity was confirmed at both group and subject level and using two different measures commonly employed for representational similarity analyses. Likewise, this difference between activation and connectivity profile was replicated using a different method for estimating task-related connectivity (i.e., PPI). Taken altogether, these results fall in line with dynamical systems perspectives of the brain that suggest tasks are separately represented at both the node (voxel or region) and edge (connectivity) levels.

How is it possible that similar patterns of brain activation arise from dissimilar network architectures? Even though our study did not enable us to directly address this question, our results provide intriguing hints that the underlying mechanisms responsible for this take place at the connectivity level. It is important to keep in mind that functional connectivity is, itself, a dynamic signal. Even though intrinsic networks, like those measured in resting-state conditions, are thought to reflect underlying structural (or at least static) networks (Hagmann et al., 2008; Honey et al., 2009; Cole et al., 2014, 2016), the reality is that correlations 
in the hemodynamic BOLD response reflect highly flexible network states. Indeed, it is well known that these networks can flexibly reconfigure in order to adapt to contextual changes (Spielberg et al., 2015), so one could expect a similar behavior even when these contextual changes do not produce a significant deviation in the brain response. In our case, network differences between tasks are not large (i.e., they are not completely independent networks), albeit significantly larger than those from brain activation patterns. Nevertheless, it is expected that these differences become more pronounced as the scale of the interacting components increase, e.g., at the neuronal level (Prinz et al., 2004; Hooper, 2004; Cropper et al., 2016). It is worth pointing out that while network profiles do differ more between tasks than activation profiles, we still observed a modest degree of commonality in network architecture across tasks as well. This similarity was mostly found in visual and motor networks that are reliably associated with sensory processing and motor planning. While motor planning constraints were identical across tasks (i.e., both involved button presses with the same hand and fingers), the visual stimuli were quite different (see Fig. 1). This suggests that the between-task dissimilarities in network profiles reflect differences in how sensory information is used during action selection, after sensory representations are formed, rather than simple bottom-up effects driven by the stimulus differences between the Stroop task and MSIT.

One natural follow-up question is how the edge time series responses compare to other approaches for addressing task-based connectivity like PPI. We have shown that, even though PPI arrives at the same conclusion as the edge time series method, the connectivity profiles obtained from both approaches were not perfectly identical. While the edge time series straightforwardly represents measures of (instantaneous) functional connectivity, PPI was designed to assess effective connectivity (Friston et al., 1997; Friston, 2011). Thus, in order to enable the comparison between both approaches in our study, PPI estimates were symmetrized, so we speculate that part of these differences may come from this operation. A full comparison with other common methods for task-related connectivity, such as correlational PPI (Fornito et al., 2012) or beta series correlations (Rissman et al., 2004), could yield both interesting differences and show areas of robustness in network profiles. However, this is well beyond the scope of this work.

While our findings here provide strong evidence in support of the dynamical systems model, some caveats must be considered. First, since connectivity matrices become computationally intractable at the voxellevel, and in order to maintain both activation and connectivity measures with the same spatial resolution, 
we opted to perform all analyses at the region level, using a predefined parcellation template. This obviously introduces some degree of anatomically bounded spatial smoothing in the data, which may be contributing to inflating the similarities in both task-related activation and connectivity between tasks. While smoothing would be problematic if we were interested in null hypotheses tests on spatial profiles (Markello and Misic, 2020), the analysis used here does not rely on such spatial hypothesis testing. Thus, this region-level approach does not invalidate the main conclusions of our study that similarity in the topology of activation profiles does not perfectly associate with similarity in network architecture. A second possible limitation is our choice to regress out the global signal as a preprocessing step. Including this preprocessing step, or not, is still somewhat controversial and it appears that both options reveal complementary information (Murphy and Fox, 2017). We opted to include it to further remove any residual noise from the signal. Finally, one might question whether the BOLD time series first needed to be deconvolved with the hemodynamic response function prior to estimating the edge time series. It has been argued that deconvolution in blockdesign tasks, like our Stroop task and MSIT, may not be necessary (Di and Biswal, 2017; Di et al., 2020). However, it is important to point out that while changing the choices in the preprocessing and analysis steps may lead to nuanced differences in certain aspects of our results, none of these potential limitations would likely change the primary conclusion we have drawn from our observations.

Regardless of these limitations, our results clearly illustrate that important aspects of task representations are encoded in the connectivity between regions. This is unique from the information reflected in the spatial topology of activation. Indeed, our findings bolster previous work looking at informational connectivity (Coutanche and Thompson-Schill, 2013), which highlights the information value of connectivity in understanding task representations. Further work should dig deeper into the high dimensional relationships between localized activation and global connectivity dynamics when trying to understand the nature of representations int he brain.

\section{Code and data availability}

The code used to generate all the analyses and results can be found in https://github.com/ CoAxLab/cofluctuating-task-connectivity. 


\section{References}

Abraham A, Pedregosa F, Eickenberg M, Gervais P, Mueller A, Kossaifi J, Gramfort A, Thirion B, Varoquaux G (2014) Machine learning for neuroimaging with scikit-learn. Frontiers in Neuroinformatics 8.

Anders S, Lotze M, Erb M, Grodd W, Birbaumer N (2004) Brain activity underlying emotional valence and arousal: A response-related fMRI study. Human Brain Mapping 23:200-209.

Banich MT, Milham MP, Atchley RA, Cohen NJ, Webb A, Wszalek T, Kramer AF, Liang ZP, Barad V, Gullett D, Shah C, Brown C (2000a) Prefrontal regions play a predominant role in imposing an attentional 'set': evidence from fMRI. Cognitive Brain Research 10:1-9.

Banich MT, Milham MP, Atchley R, Cohen NJ, Webb A, Wszalek T, Kramer AF, Liang ZP, Wright A, Shenker J, Magin R (2000b) fMRI studies of stroop tasks reveal unique roles of anterior and posterior brain systems in attentional selection. Journal of Cognitive Neuroscience 12:988-1000.

Behrens TE, Sporns O (2012) Human connectomics. Current opinion in neurobiology 22:144-153.

Bertolero MA, Yeo BTT, D'Esposito M (2015) The modular and integrative functional architecture of the human brain. Proceedings of the National Academy of Sciences 112:E6798-E6807.

Bihan DL, Turner R, Zeffiro TA, Cuenod CA, Jezzard P, Bonnerot V (1993) Activation of human primary visual cortex during visual recall: a magnetic resonance imaging study. Proceedings of the National Academy of Sciences 90:11802-11805.

Binder JR, Frost JA, Hammeke TA, Cox RW, Rao SM, Prieto T (1997) Human brain language areas identified by functional magnetic resonance imaging. The Journal of Neuroscience 17:353-362.

Bush G, Shin LM (2006) The multi-source interference task: an fMRI task that reliably activates the cingulo-frontal-parietal cognitive/attention network. Nature Protocols 1:308-313.

Churchland MM, Cunningham JP, Kaufman MT, Foster JD, Nuyujukian P, Ryu SI, Shenoy KV (2012) Neural population dynamics during reaching. Nature 487:51-56. 
Cole MW, Bassett DS, Power JD, Braver TS, Petersen SE (2014) Intrinsic and task-evoked network architectures of the human brain. Neuron 83:238-251.

Cole MW, Ito T, Bassett DS, Schultz DH (2016) Activity flow over resting-state networks shapes cognitive task activations. Nature neuroscience 19:1718-1726.

Cole MW, Ito T, Schultz D, Mill R, Chen R, Cocuzza C (2019) Task activations produce spurious but systematic inflation of task functional connectivity estimates. NeuroImage 189:1-18.

Coutanche MN, Thompson-Schill SL (2013) Informational connectivity: identifying synchronized discriminability of multi-voxel patterns across the brain. Frontiers in human neuroscience 7:15.

Cropper EC, Dacks AM, Weiss KR (2016) Consequences of degeneracy in network function. Current Opinion in Neurobiology 41:62-67.

Crossley NA, Mechelli A, Vertes PE, Winton-Brown TT, Patel AX, Ginestet CE, McGuire P, Bullmore ET (2013) Cognitive relevance of the community structure of the human brain functional coactivation network. Proceedings of the National Academy of Sciences 110:11583-11588.

Deng Y, Wang X, Wang Y, Zhou C (2018) Neural correlates of interference resolution in the multi-source interference task: a meta-analysis of functional neuroimaging studies. Behavioral and Brain Functions 14.

Di X, Biswal BB (2017) Psychophysiological interactions in a visual checkerboard task: Reproducibility, reliability, and the effects of deconvolution. Frontiers in Neuroscience 11.

Di X, Reynolds RC, Biswal BB (2017) Imperfect (de)convolution may introduce spurious psychophysiological interactions and how to avoid it. Human Brain Mapping 38:1723-1740.

Di X, Zhang Z, Biswal BB (2020) Understanding psychophysiological interaction and its relations to beta series correlation. Brain Imaging and Behavior 15:958-973.

Ejaz N, Hamada M, Diedrichsen J (2015) Hand use predicts the structure of representations in sensorimotor cortex. Nature neuroscience 18:1034-1040.

Esteban O, Markiewicz CJ, Blair RW, Moodie CA, Isik AI, Erramuzpe A, Kent JD, Goncalves M, DuPre 
E, Snyder M, Oya H, Ghosh SS, Wright J, Durnez J, Poldrack RA, Gorgolewski KJ (2018) fMRIPrep: a robust preprocessing pipeline for functional MRI. Nature Methods 16:111-116.

Faskowitz J, Esfahlani FZ, Jo Y, Sporns O, Betzel RF (2020) Edge-centric functional network representations of human cerebral cortex reveal overlapping system-level architecture. Nature Neuroscience 23:1644-1654.

Feinberg TE, Farah MJ (2006) A historical perspective on cognitive neuroscience. .

Finn ES, Shen X, Scheinost D, Rosenberg MD, Huang J, Chun MM, Papademetris X, Constable RT (2015) Functional connectome fingerprinting: identifying individuals using patterns of brain connectivity. Nature Neuroscience 18:1664-1671.

Fodor JA (1983) The Modularity of Mind: An Essay on Faculty Psychology Cambridge, MA: MIT Press.

Fornito A, Harrison BJ, Zalesky A, Simons JS (2012) Competitive and cooperative dynamics of largescale brain functional networks supporting recollection. Proceedings of the National Academy of Sciences 109:12788-12793.

Friston KJ (2011) Functional and effective connectivity: A review. Brain Connectivity 1:13-36.

Friston KJ, Holmes AP, Worsley KJ, Poline JP, Frith CD, Frackowiak RS (1994) Statistical parametric maps in functional imaging: a general linear approach. Human brain mapping 2:189-210.

Friston KJ, Williams S, Howard R, Frackowiak RSJ, Turner R (1996) Movement-related effects in fMRI time-series. Magnetic Resonance in Medicine 35:346-355.

Friston K, Buechel C, Fink G, Morris J, Rolls E, Dolan R (1997) Psychophysiological and modulatory interactions in neuroimaging. NeuroImage 6:218-229.

Gelder TV (1995) What might cognition be, if not computation? Journal of Philosophy 92:345-381.

Gianaros PJ, Kraynak TE, Kuan DCH, Gross JJ, McRae K, Hariri AR, Manuck SB, Rasero J, Verstynen TD (2020) Affective brain patterns as multivariate neural correlates of cardiovascular disease risk 15:1034-1045. 
Hagmann P, Cammoun L, Gigandet X, Meuli R, Honey CJ, Wedeen VJ, Sporns O (2008) Mapping the structural core of human cerebral cortex. PLoS biology 6:e159.

Honey CJ, Sporns O, Cammoun L, Gigandet X, Thiran JP, Meuli R, Hagmann P (2009) Predicting human resting-state functional connectivity from structural connectivity. Proceedings of the National Academy of Sciences 106:2035-2040.

Hooper SL (2004) Multiple routes to similar network output. Nature Neuroscience 7:1287-1288.

Kriegeskorte N, Mur M, Ruff DA, Kiani R, Bodurka J, Esteky H, Tanaka K, Bandettini PA (2008) Matching categorical object representations in inferior temporal cortex of man and monkey. Neuron 60:1126-1141.

Lindquist MA, Geuter S, Wager TD, Caffo BS (2019) Modular preprocessing pipelines can reintroduce artifacts into fMRI data. Human Brain Mapping 40:2358-2376.

MacDonald AW (2000) Dissociating the role of the dorsolateral prefrontal and anterior cingulate cortex in cognitive control. Science 288:1835-1838.

Markello RD, Misic B (2020) Comparing spatial null models for brain maps .

McLaren DG, Ries ML, Xu G, Johnson SC (2012) A generalized form of context-dependent psychophysiological interactions (gPPI): A comparison to standard approaches. NeuroImage 61:1277-1286.

Milham M, Banich M, Webb A, Barad V, Cohen N, Wszalek T, Kramer A (2001) The relative involvement of anterior cingulate and prefrontal cortex in attentional control depends on nature of conflict. Cognitive Brain Research 12:467-473.

Murphy K, Fox MD (2017) Towards a consensus regarding global signal regression for resting state functional connectivity MRI. NeuroImage 154:169-173.

Oby ER, Golub MD, Hennig JA, Degenhart AD, Tyler-Kabara EC, Byron MY, Chase SM, Batista AP (2019) New neural activity patterns emerge with long-term learning. Proceedings of the National Academy of Sciences 116:15210-15215.

Porro CA, Francescato MP, Cettolo V, Diamond ME, Baraldi P, Zuiani C, Bazzocchi M, di Prampero PE 
(1996) Primary motor and sensory cortex activation during motor performance and motor imagery: A functional magnetic resonance imaging study. The Journal of Neuroscience 16:7688-7698.

Posner M, Petersen S, Fox P, Raichle M (1988) Localization of cognitive operations in the human brain. Science 240:1627-1631.

Power JD, Barnes KA, Snyder AZ, Schlaggar BL, Petersen SE (2012) Spurious but systematic correlations in functional connectivity MRI networks arise from subject motion. NeuroImage 59:2142-2154.

Prinz AA, Bucher D, Marder E (2004) Similar network activity from disparate circuit parameters. Nature Neuroscience 7:1345-1352.

Rissman J, Gazzaley A, D’Esposito M (2004) Measuring functional connectivity during distinct stages of a cognitive task. NeuroImage 23:752-763.

Russo AA, Khajeh R, Bittner SR, Perkins SM, Cunningham JP, Abbott LF, Churchland MM (2020) Neural trajectories in the supplementary motor area and motor cortex exhibit distinct geometries, compatible with different classes of computation. Neuron 107:745-758.

Sadtler PT, Quick KM, Golub MD, Chase SM, Ryu SI, Tyler-Kabara EC, Byron MY, Batista AP (2014) Neural constraints on learning. Nature 512:423-426.

Shen X, Tokoglu F, Papademetris X, Constable R (2013) Groupwise whole-brain parcellation from restingstate fMRI data for network node identification. NeuroImage 82:403-415.

Sheu LK, Jennings JR, Gianaros PJ (2012) Test-retest reliability of an fMRI paradigm for studies of cardiovascular reactivity. Psychophysiology 49:873-884.

Spielberg JM, Miller GA, Heller W, Banich MT (2015) Flexible brain network reconfiguration supporting inhibitory control. Proceedings of the National Academy of Sciences 112:10020-10025.

Sporns O (2013) Network attributes for segregation and integration in the human brain. Current Opinion in Neurobiology 23:162-171.

Stroop JR (1935) Studies of interference in serial verbal reactions. Journal of Experimental Psychology 18:643-662. 
bioRxiv preprint doi: https://doi.org/10.1101/2021.11.27.470015; this version posted November 27, 2021. The copyright holder for this preprint (which was not certified by peer review) is the author/funder, who has granted bioRxiv a license to display the preprint in perpetuity. It is made available under aCC-BY-NC-ND 4.0 International license.

Yeo BTT, Krienen FM, Eickhoff SB, Yaakub SN, Fox PT, Buckner RL, Asplund CL, Chee MW (2014)

Functional specialization and flexibility in human association cortex. Cerebral Cortex 25:3654-3672.

Zamani Esfahlani F, Jo Y, Faskowitz J, Byrge L, Kennedy DP, Sporns O, Betzel RF (2020) High-amplitude cofluctuations in cortical activity drive functional connectivity. Proceedings of the National Academy of Sciences 117:28393-28401. 


\section{Supporting information}
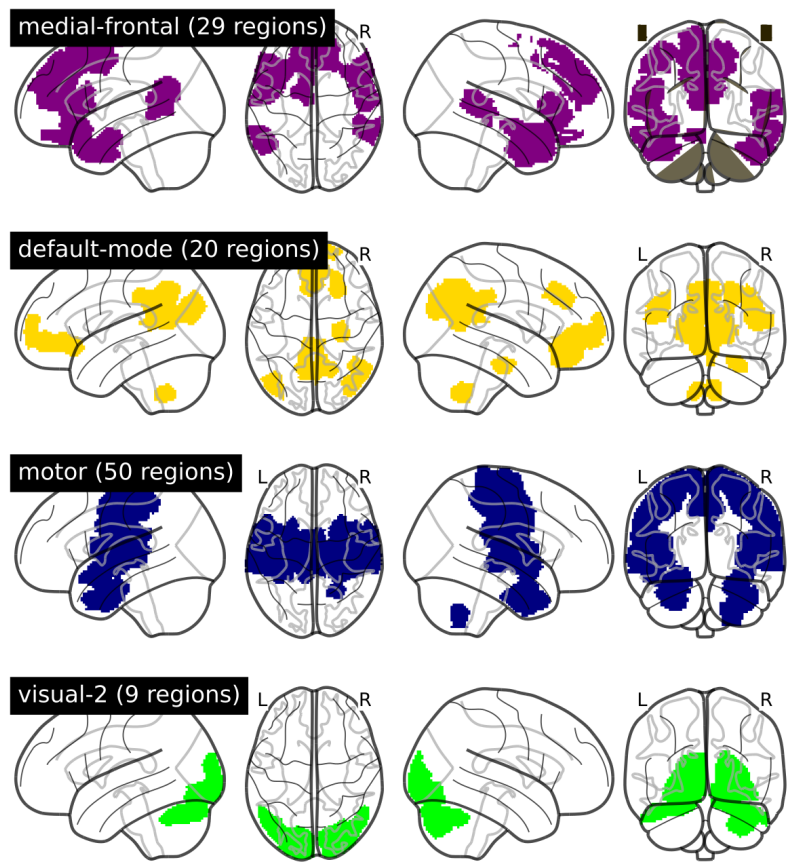
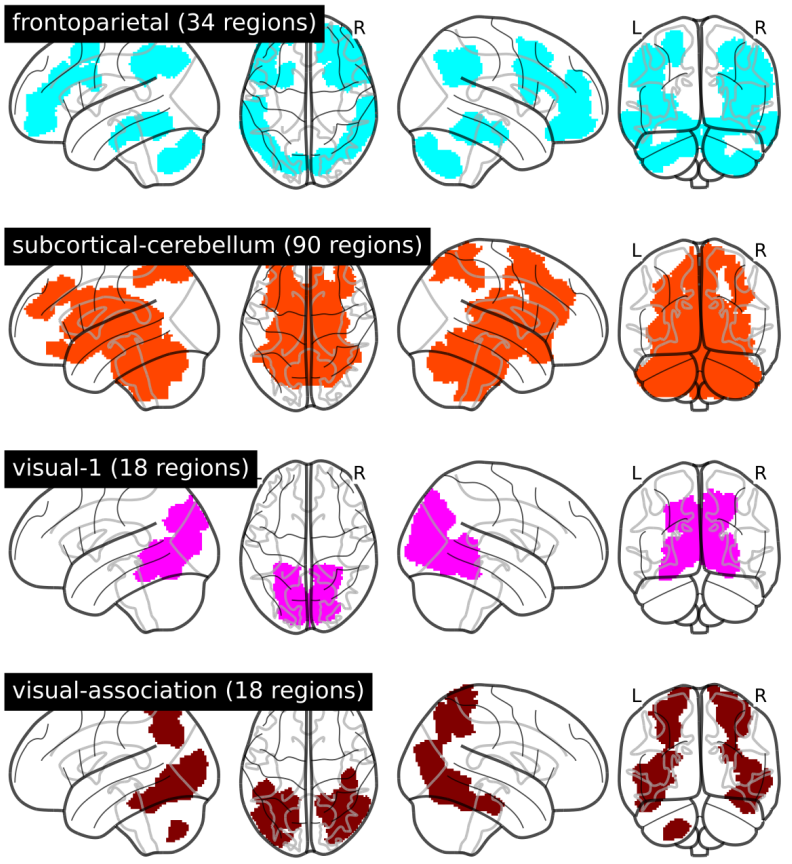

Figure S1: Brain Atlas. The 268 region parcellation used in our study. Each of these regions was further assigned to one specific intrinsic connectivity network (Finn et al., 2015); namely, Medial-Frontal, Frontoparietal, Default-mode, Subcortical-Cerebellum, Motor, Visual-1, Visual-2 and Visual-Association. 

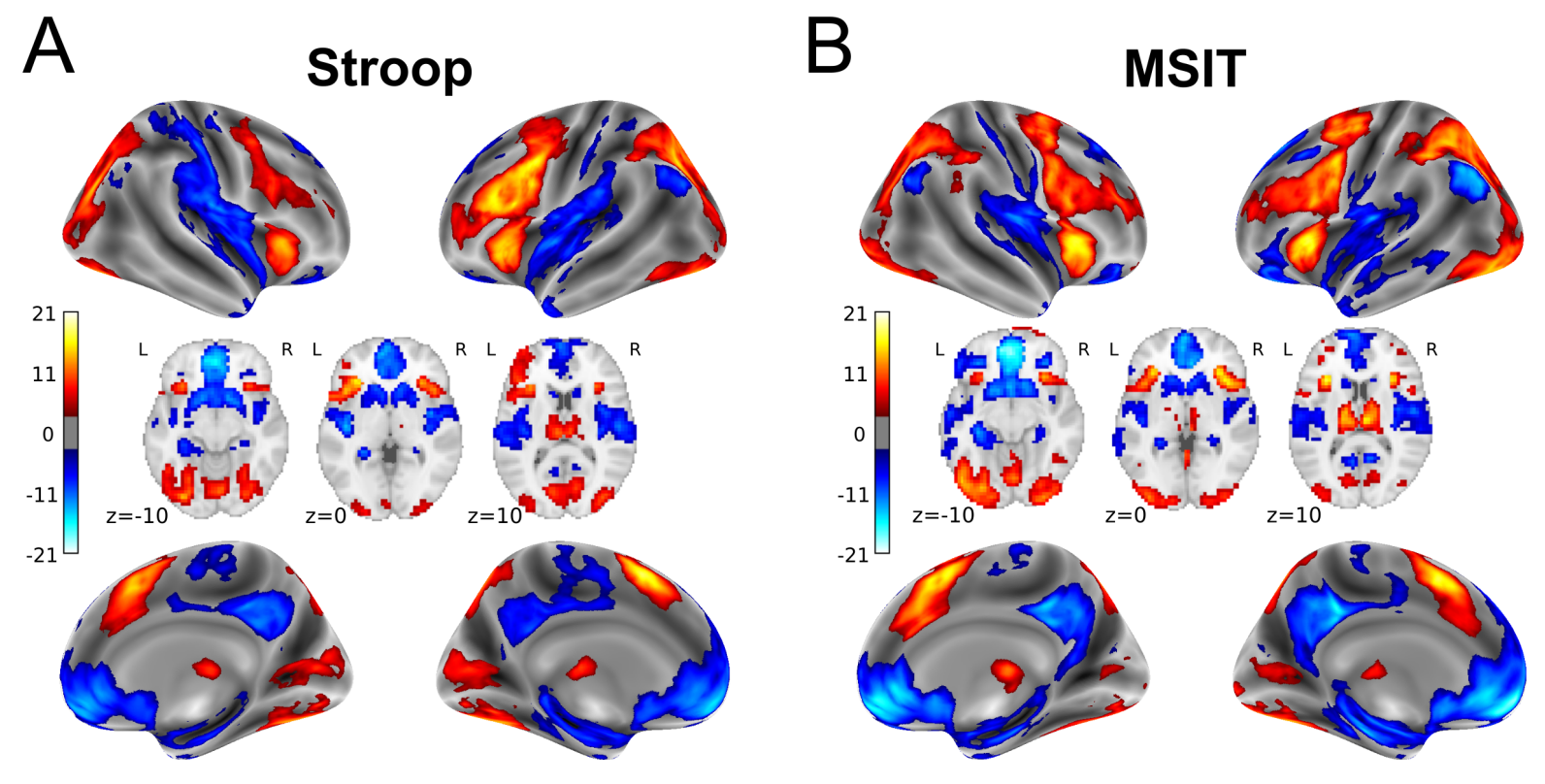

Figure S2: Group-level activation maps. For both Stroop task (A) and MSIT (B), the group-level incongruent-vs-congruent t-stat maps. Thus, red colors display higher BOLD activity during incongruent trials compared to congruent trials, whereas blue colors represent the other way around. 


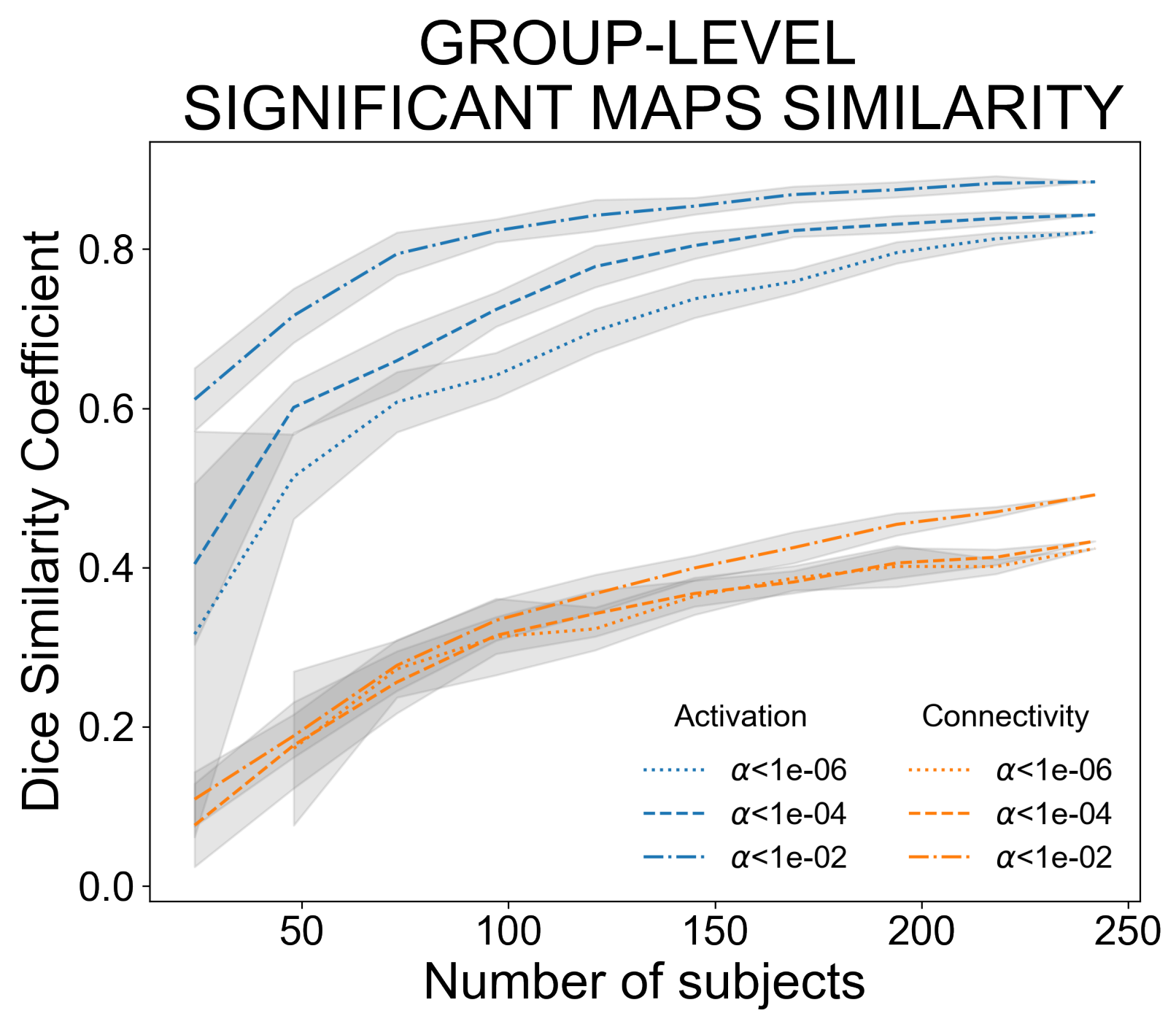

Figure S3: Overlapping analysis of activation and connectivity profiles. Overlapping analysis between tasks of both activation (in blue) and connectivity (in orange) t-stat maps using the dice similarity coefficient. Statistical maps were binarized according to whether each t-stat was significant or not under several thresholds $\alpha$. 


\section{Similarity of task-based connectivity maps between Stroop and MSIT}

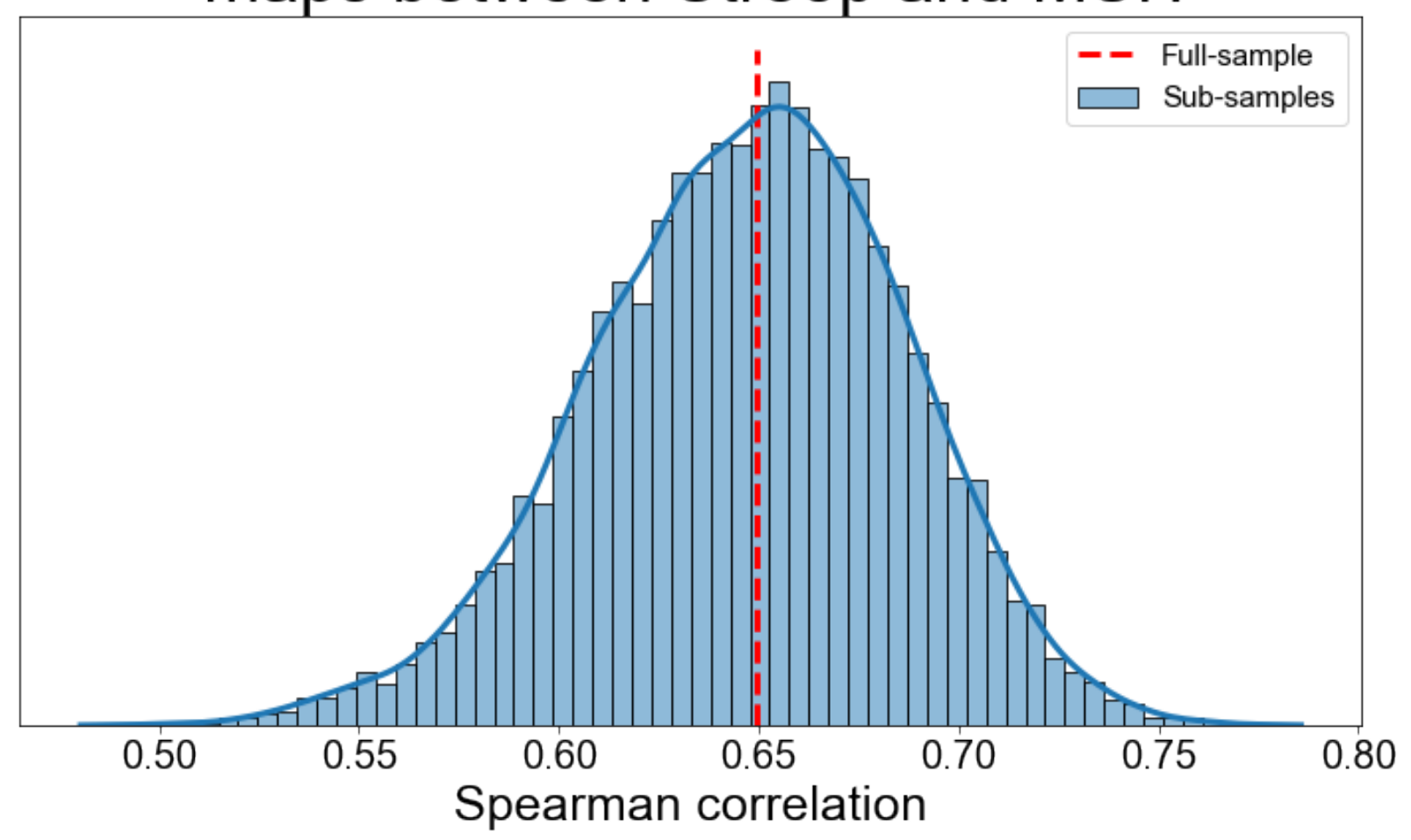

Figure S4: Distribution of between-tasks similarity in connectivity profiles. By taking subsamplings in the number of links to be the same of regions (268), the distribution of spearman correlations $\rho$ between MSIT and Stroop connectivity profiles. 


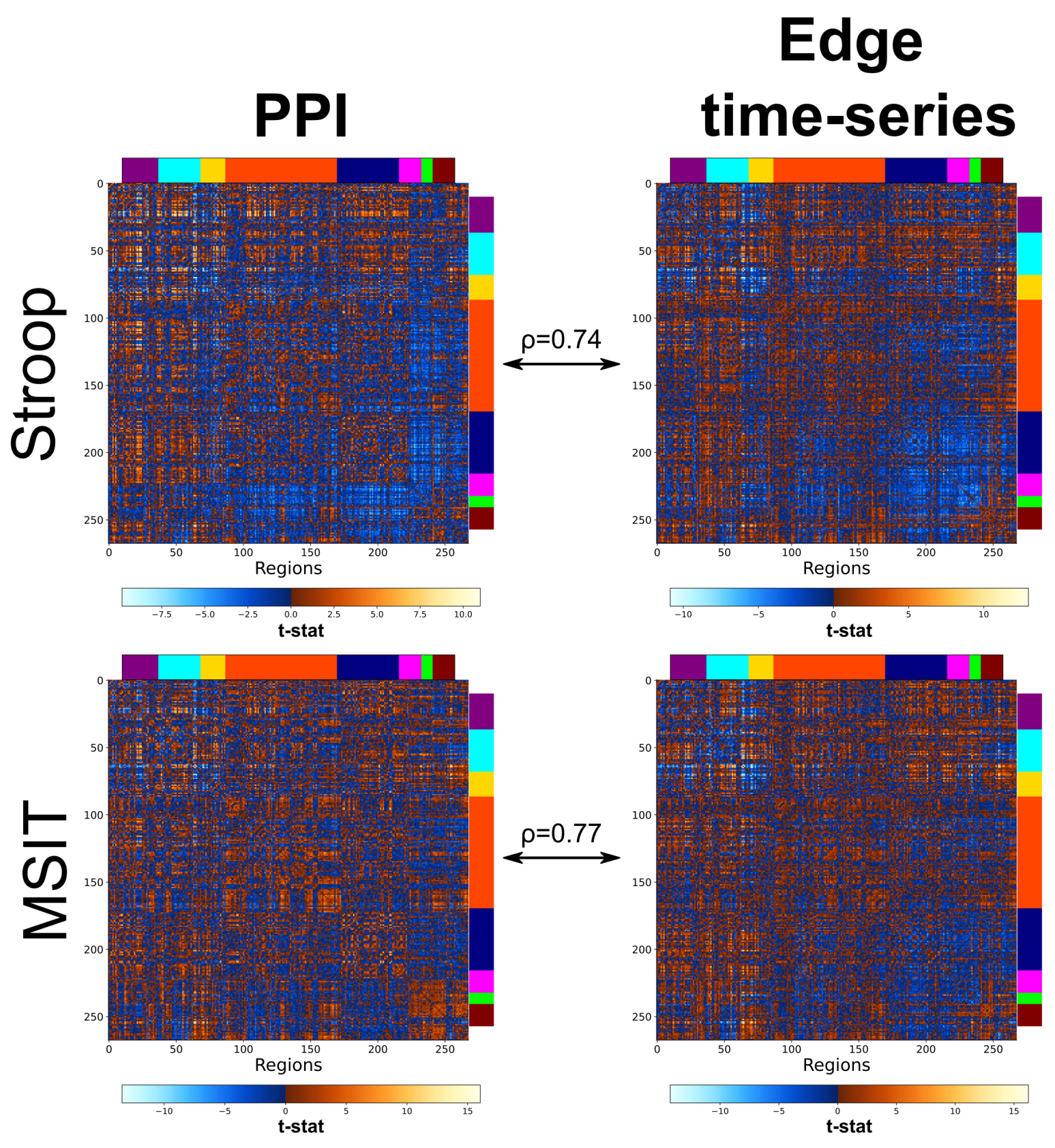

Figure S5: PPI-based connectivity contrast maps. On the left, the PPI-based incongruent-vs-congruent connectivity maps, depicted as matrices, for both Stroop and MSIT. On the right, the same maps using our edge-time series approach. A Spearman correlation $\rho$ was also computed to compare both methods within tasks. 